\title{
Liver Aminotransferases Are Elevated with Rhabdomyolysis in the Absence of Significant Liver Injury
}

\author{
Kathryn Weibrecht • Matthew Dayno $\cdot$ Chad Darling • \\ Steven B. Bird
}

Published online: 21 April 2010

(C) American College of Medical Toxicology 2010

\begin{abstract}
Rhabdomyolysis is an uncommon finding in the emergency department. However, the clinical implications of rhabdomyolysis are important, with a significant minority of patients developing acute renal failure and multiorgan failure. When present, the cause of elevated aminotransferases in the setting of rhabdomyolysis is often unclear. We sought to determine the incidence of abnormal aminotransferases (defined as aspartate aminotransferase (AST) or alanine aminotransferase (ALT) $>40 \mathrm{U} / \mathrm{L}$ ) in the setting of rhabdomyolysis and how the aminotransferases decrease relative to the creatine phosphokinase (CPK) concentration as rhabdomyolysis resolves. A retrospective chart review of 215 cases of rhabdomyolysis with $\mathrm{CPK}$ of $\geq 1,000 \mathrm{U} / \mathrm{L}$ was performed. The incidence of an abnormal AST in the setting of rhabdomyolysis was $93.1 \%$ (95\% confidence interval, $88.7 \%$ to $95.8 \%$ ). An abnormal ALT was much less common and found in $75.0 \%$ (95\% confidence interval, $68.7 \%$ to $80.2 \%)$ of patients with a CPK of $\geq 1,000 \mathrm{U} / \mathrm{L}(p<0.0001)$. In only one instance was the ALT $>40 \mathrm{U} / \mathrm{L}$ while the AST was $<40 \mathrm{U} / \mathrm{L}$. Furthermore, AST concentrations (and not ALT) fall in parallel with CPK during the first 6 days of hospitalization for patients with rhabdomyolysis. Aminotransferase abnormalities, particularly AST, are common in the setting of rhabdomyolysis. AST concentrations decrease
\end{abstract}

K. Weibrecht $\cdot$ S. B. Bird $(\bowtie)$

Division of Medical Toxicology,

University of Massachusetts Medical School,

55 Lake Avenue North,

Worcester, MA, USA

e-mail: Steven.bird@umassmemorial.org

M. Dayno · C. Darling

Department of Emergency Medicine,

University of Massachusetts Medical School,

55 Lake Avenue North,

Worcester, MA, USA in parallel to CPK, suggesting skeletal muscle may be a significant source of AST elevation in these patients.

Keywords Aspartate aminotransferase - Alanine aminotransferase $\cdot$ Rhabdomyolysis

\section{Introduction}

Background and Importance

Rhabdomyolysis (rhabdo-striated, myo-muscle, and lysis - disintegration) is an uncommon clinical condition first described in English victims of crush injuries suffered during the German military blitzkrieg bombings [1]. Since then, the metabolic and end organ consequences of rhabdomyolysis have been fully recognized, although best treatment strategies are not well defined.

Rhabdomyolysis begins with simple muscle injury. This injury is generally due to exertion, medication effects, trauma, prolonged immobilization, illicit drug abuse, or infection [2]. If this muscle injury is severe enough, cellular proteins such as creatinine phosphokinase (CPK) accumulate in the circulation and eventually are deposited in renal tubules and other organs. A minority of patients with rhabdomyolysis develop acute renal failure (due to acute tubular necrosis) and multiorgan failure [3, 4].

The clinical history obtained from patients with rhabdomyolysis is often limited, particularly in the elderly or when there has been recent substance abuse. We have encountered a number of cases presenting with elevated aminotransferases (aspartate aminotransferase [AST] and alanine aminotransferase [ALT]) in the setting of oral opiateacetaminophen combination abuse, rhabdomyolysis, and a minimally detectable acetaminophen concentration. In this 
scenario, it is difficult to determine if the elevated aminotransferases are due to acetaminophen ingestion or another cause. All of these cases were treated with $\mathrm{N}$-acetylcysteine and the aminotransferases quickly returned to the normal range, but we suspect that the aminotransferase abnormalities in these patients may not be solely a result of acetaminophen-mediated liver injury.

Goals of this Investigation

This investigation was undertaken in order to obtain additional insight into the potential cause of AST and ALT elevations in patients with rhabdomyolysis. Specifically, we sought to describe the incidence and extent of abnormal AST or ALT concentrations in the setting of rhabdomyolysis and determine the relationship between CPK concentrations and aminotransferase concentrations in these patients.

\section{Materials and Methods}

\section{Study Design and Setting}

We performed a retrospective chart review and descriptive case series study of hospitalized patients with an ICD-9 code of rhabdomyolysis from September 2003 to September 2007. The study was considered exempt from IRB review by our institutional human subjects research office. The study was conducted in an urban, tertiary care, university teaching hospital in central Massachusetts with annual emergency department patient visits of approximately 85,000, an emergency medicine residency, a toxicology fellowship, and a toxicology consult service.

\section{Selection of Participants}

Inclusion criteria included at least one serum CPK concentration of $\geq 1000 \mathrm{IU} / \mathrm{mL}$, at least one AST or ALT measurement, and age $\geq 18$. A CPK of $\geq 1,000 \mathrm{IU} / \mathrm{mL}$ was chosen because that value appears most often in the literature in discussions of clinically meaningful rhabdomyolysis [2, 5]. Exclusion criteria included diagnoses of acetaminophen overdose by history or laboratory confirmation, myocardial infarction, the current use of a statin drug, the development of rhabdomyolysis while hospitalized, acute or chronic hepatitis B or C infection, or chronically elevated aminotransferases (as determined from primary care or subspecialist's notes). Because we were interested in the acute aminotransferase and CPK concentrations during rhabdomyolysis, we abstracted data from the first 6 days of hospitalization.
Methods of Measurement and Data Collection

Data were abstracted in a structured format by two of the authors (MD, KW) and subsequently entered into a customized data form on an Excel spreadsheet (Microsoft Corp, Redmond, WA, USA) using described methodologies [6]. Demographic data collected included age, gender, admission/discharge dates, duration of hospital stay, admission medication use, and discharge diagnoses. Key lab values (up to and including the sixth hospital day) obtained included serum CPK, AST, ALT, creatinine, international normalized ratio (INR), and troponin. All laboratory samples were analyzed using a Unicell DXC 800 clinical analyzer (Beckman Coulter Inc, Brea, CA, USA).

\section{Primary Data Analysis}

Data were analyzed and graphed using either GraphPad Prism for Mac (GraphPad Software, Inc., La Jolla, CA, USA) or Excel. Descriptive statistics and aminotransferase means and medians were calculated. The peak aminotransferase concentrations were analyzed for normality with the D'Agostino-Pearson omnibus test and were stratified according to CPK (CPK $<10,000$, CPK 10,000-20,000, or $\mathrm{CPK}>20,000)$ and subsequently compared by the KruskalWallis test.

\section{Results}

A total of 673 patients were identified. From the 673 patients, several were excluded as follows: 223 did not have a CPK measurement $\geq 1,000 ; 125$ had no AST or ALT measured during hospitalization; 53 were on a statin therapy; 34 had myocardial infarction; 11 had known hepatitis infections; eight were diagnosed with acetaminophen poisoning; one had chronically elevated aminotransferases; one patient could not be identified from medical records; one patient was present twice in the database; and one patient was less than 18 years old. Therefore, a total of 215 patients remained for analyses.

Patient demographics are presented in Table 1. Most patients had three or more CPK measurements during the 6 days; only 36 patients $(16.9 \%)$ had less than three CPK measurements. Mean CPK, AST, and ALT measurements for all 215 patients for hospital days 0 through 5 are presented in Fig. 1. Mean CPK and AST concentrations fell in parallel to each other, while ALT concentrations fell more slowly and not in parallel with either CPK or AST. The mean CPK fell by $74.5 \%$ from day 0 to 5 while the mean AST values fell by $78.9 \%$. However, mean ALT concentrations decreased by just $57.8 \%$ during that time. 
Table 1 Patient demographics

\begin{tabular}{lc}
\hline Number & 215 \\
Age [years (mean+SD)] & $54 \pm 23$ \\
Number of Male (\%) & $130(60.5 \%)$ \\
Number with 6 CPK data (\%) & $63(29.3)$ \\
Number with 5 CPK data (\%) & $43(20.0)$ \\
Number with 4 CPK data (\%) & $39(18.1)$ \\
Number with 3 CPK data (\%) & $35(16.3)$ \\
Number with 2 CPK data (\%) & $28(13.0)$ \\
Number with 1 CPK datum (\%) & $7(3.2)$ \\
6-day mortality [number (\%)] & $7(3.2)$ \\
Number requiring dialysis (\%) & $11(5.1)$ \\
Number on Coumadin (\%) & $12(5.6)$ \\
\hline
\end{tabular}

Of the 215 patients, $15 \mathrm{did}$ not have an AST $>40 \mathrm{U} / \mathrm{L}$, yielding an incidence of an abnormal AST in the setting of rhabdomyolysis of $93.0 \%$ (95\% confidence interval, $88.7 \%$ to $95.8 \%)$. An abnormal ALT was much less common and found in $161(75.0 \%)(95 \%$ confidence interval, $68.7 \%$ to $80.2 \%$ ) of patients with a CPK of $\geq 1,000 \mathrm{U} / \mathrm{L}$. In only one instance was the ALT $>40 \mathrm{U} / \mathrm{L}$ and the AST $<40 \mathrm{U} / \mathrm{L}$. The peak CPK and AST or ALT values for each of the 215 patients are presented in Figs. 2 and 3, respectively.

The peak aminotransferase concentrations were not normally distributed. Therefore, when patients were stratified according to peak CPK $(\mathrm{CPK}<10,000, \mathrm{CPK}$ $10,000-20,000$, or $\mathrm{CPK}>20,000$ ) and compared using the Kruskal-Wallis test, the median peak AST was found to increase linearly (Fig. 4). The three CPK groups were found to have statistically significant different medians, except for the CPK 10,000-20,000 vs CPK $>20,000$ groups. An analysis of peak ALT vs peak CPK demonstrated identical findings (Fig. 5).

The relationship between peak CPK and peak serum creatinine for all patients is presented in Fig. 6. The data are not perfectly representative of each case because the

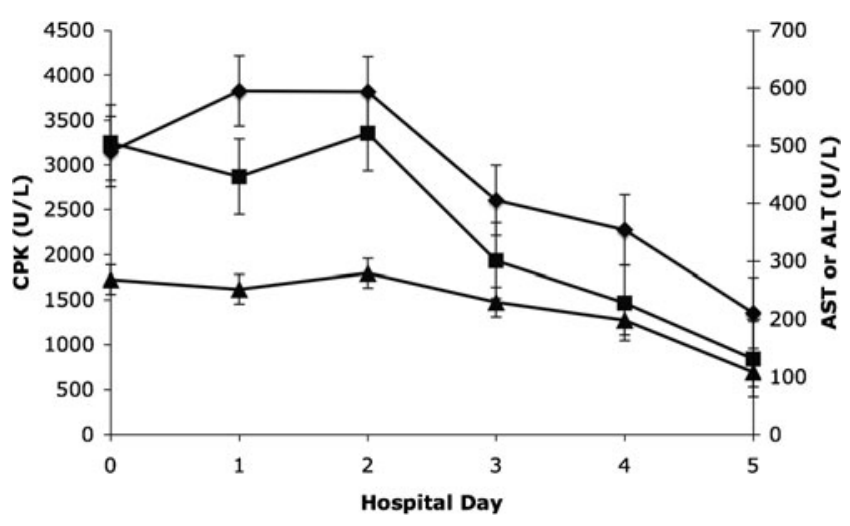

Fig. 1 Cohort mean CPK, AST, and ALT over 6 days. Filled diamond represents CPK; filled square represents $\mathrm{AST}$; and filled triangle represents ALT clinical laboratory most frequently listed the CPK concentration as " 45,100 " whenever the value exceeded 45,000 . There was not a change in laboratory methods during the study period, but rather a change in the reporting of results. Despite this bias, as evident from Fig. 6, there is a weak correlation between peak CPK and peak creatinine $\left(R^{2}=\right.$ 0.0997). Acute renal failure requiring hemodialysis occurred in 11 (5.1\%; $95 \%$ confidence interval, $2.8 \%$ to $9.0 \%$ ) of the 215 patients.

Just 141 of the 215 patients had the INR measured during the first 2 days of hospitalization. In these patients, the mean ( $\pm \mathrm{SD}$ ) INR was $1.35 \pm 0.86$. Of the $12(5.6 \%)$ patients on Coumadin at the time of presentation, their mean INR $( \pm \mathrm{SD})$ was found to be $2.2 \pm 1.1$. Excluding the patients on Coumadin, the mean INR of all patients decreased to $1.2 \pm 0.8$.

In the 15 patients who did not have any AST value $>40 \mathrm{U} / \mathrm{L}$, the mean $( \pm \mathrm{SD}) \mathrm{CPK}$ and AST values were $2,282 \pm 3,135$ and $30 \pm 7$, respectively. This CPK concentration is significantly lower than the CPK in the group with any $\mathrm{AST}>40 \mathrm{U} / \mathrm{L}(p<0.002)$.

Nine patients died within 6 days (and one suffered a cardiac arrest but was resuscitated) yielding a mortality of $4.2 \%$ (95\% confidence interval, $2.1 \%$ to $7.9 \%)$. One patient in this group was promptly made "comfort measures only" by family and had limited lab testing performed. The eight other patients in this group had a higher mean peak AST $(4,145 \pm 4,210)$, ALT $(2,197 \pm 1,933)$, CPK $(21,130 \pm$ $17,948)$, creatinine $(6.1 \pm 4.3)$, and INR $(4.5 \pm 4.0)$ values compared to survivors $(p<0.002$ for all).

\section{Limitations}

There are several limitations to this study. Firstly, like any retrospective chart review, missing data could not always be retrieved. However, this study relied on a relatively limited number of data, which could usually be accounted for by the structured chart review we performed.

A second limitation to this study is the lack of a strict diagnosis for each patient. One of the most common presenting complaints of patients in this study was "found down." This group of patients often was found to have sepsis, generalized weakness, to have used one or more drugs of abuse, or to have some other medical condition. We believe that it is impossible to assign a primary or causative diagnosis in such cases. Furthermore, some contribution to the aminotransferase abnormalities in a subset of patients (such as those with septic shock) was likely due to poor perfusion and ischemic hepatitis (shock liver). However, there are no specific criteria to prospectively define shock liver [7], and it is impossible to determine the relative contributions of rhabdomyolysis or shock liver to the observed aminotransferase abnormalities. Because of these limitations, we do not feel that even a 
Fig. 2 Scatter gram of individual peak $\mathrm{CPK}$ and peak AST values

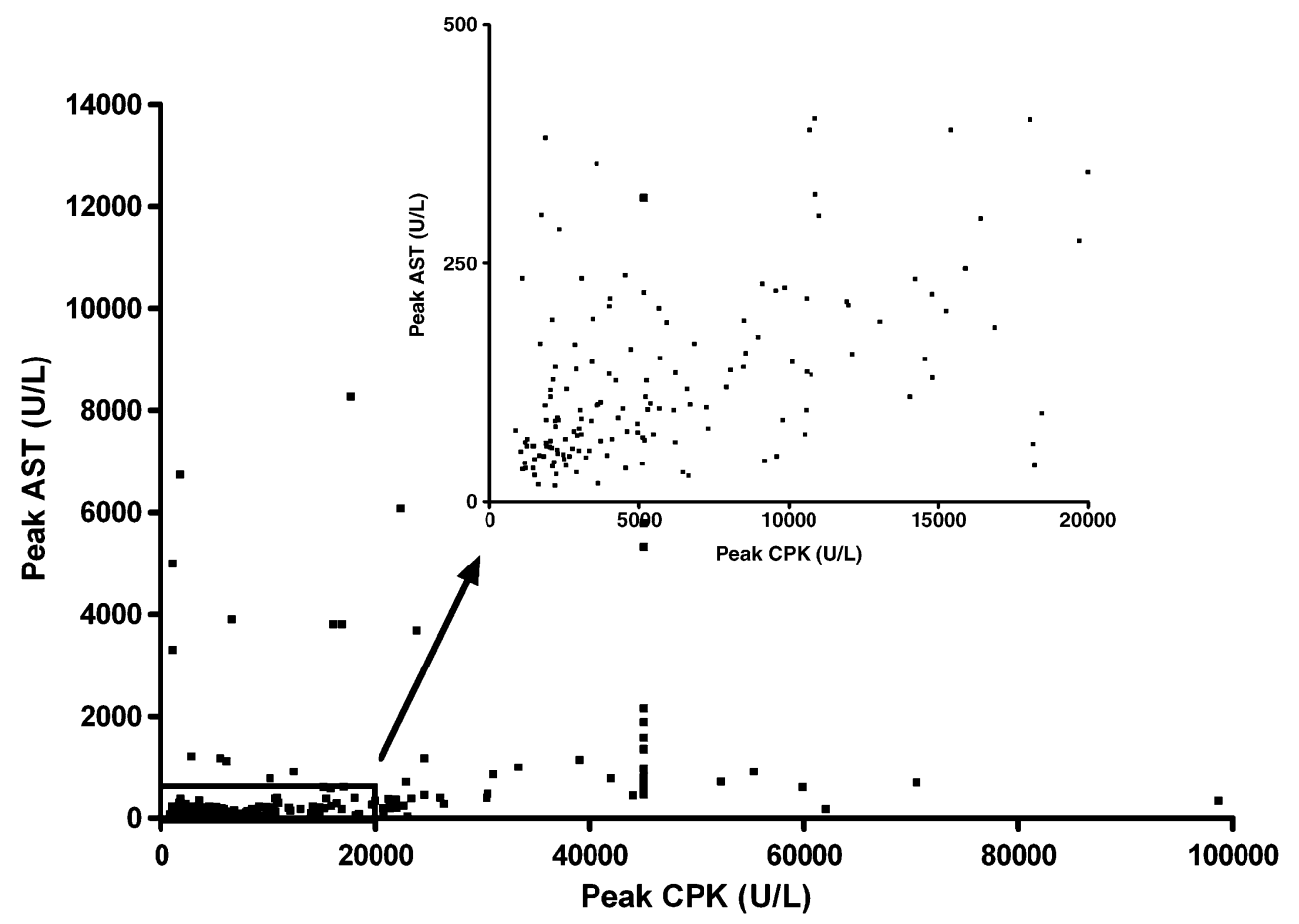

prospective study of rhabdomyolysis could adequately address the problems inherent in assigning a cause of the rhabdomyolysis or assigning the contribution of other medical conditions to the degree of elevated aminotransferases.

Interestingly, despite a diagnosis of rhabdomyolysis with abnormally elevated aminotransferases, a minority of patients did not have serial CPK or LFT measurements. While the argument could be made that such serial laboratory values would not have changed medical management, we find this interesting in light of the frequent and large volume of laboratory testing that often takes place for hospitalized patients - including an average of nearly five tests and up to $50 \mathrm{~mL}$ of blood per patient per day [8, 9]. There is likely little selection bias in our cohort of patients who had aminotransferase concentrations measured and followed. When faced with a patient with a medication
Fig. 3 Scatter gram of individual peak $\mathrm{CPK}$ and peak ALT values

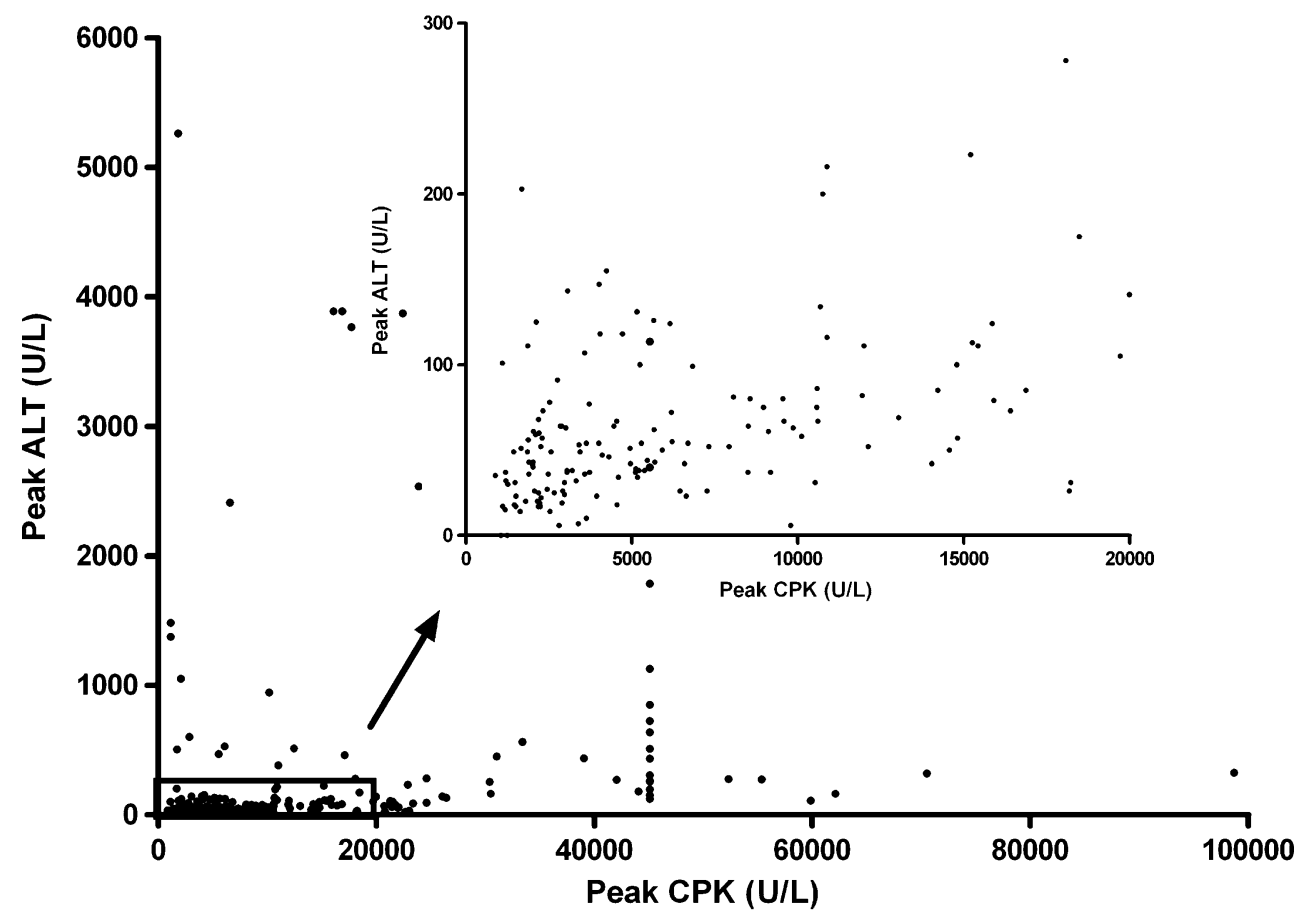




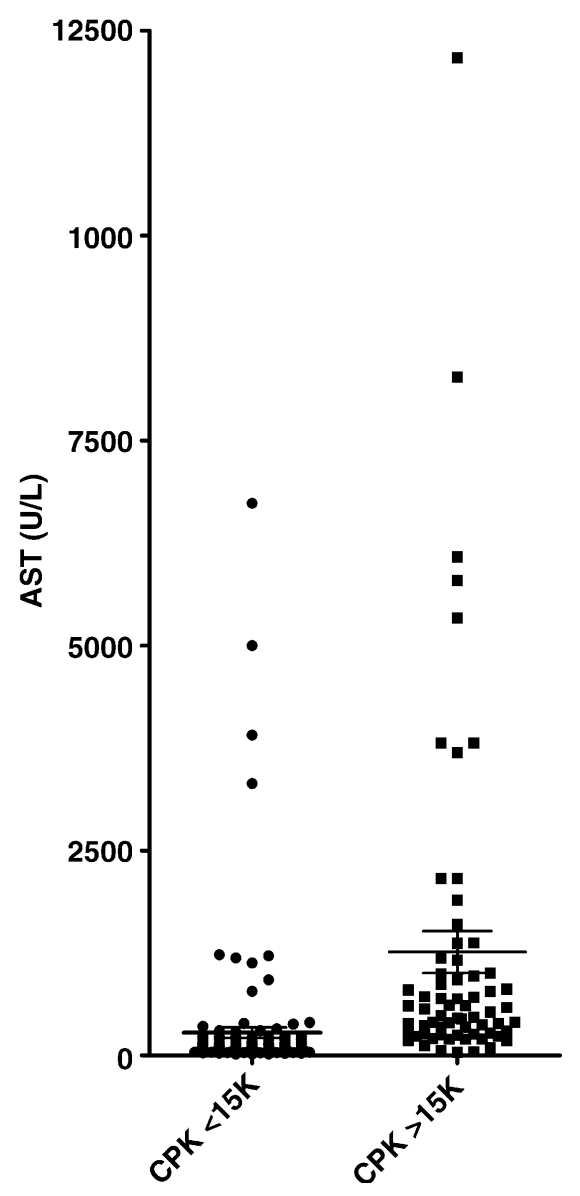

Fig. 4 Peak AST values in all patients stratified according to peak CPK value

ingestion history, a patient "found down," or similarly unclear history, it is our experience that most clinicians tend to obtain more laboratory tests. Thus, it is to be expected that these complicated patients would have more aminotransferase concentration determinations.

Additionally, blood for the laboratory tests were not always drawn at the same time and some patients had several blood draws done each day. In an effort to control for this, we collected the maximum laboratory values for each 24-h period for each patient. Lastly, the different serum clearance rates, methods of elimination, and halflives for the various serum markers add a further degree of uncertainty with regards to interpretation. Because CPK, AST, and ALT continue to be produced by organs and released into serum with rhabdomyolysis, it is impossible to calculate the half-lives of any of these biomarkers.

\section{Discussion}

Rhabdomyolysis is an uncommon, but important, clinical condition encountered in the emergency department and is

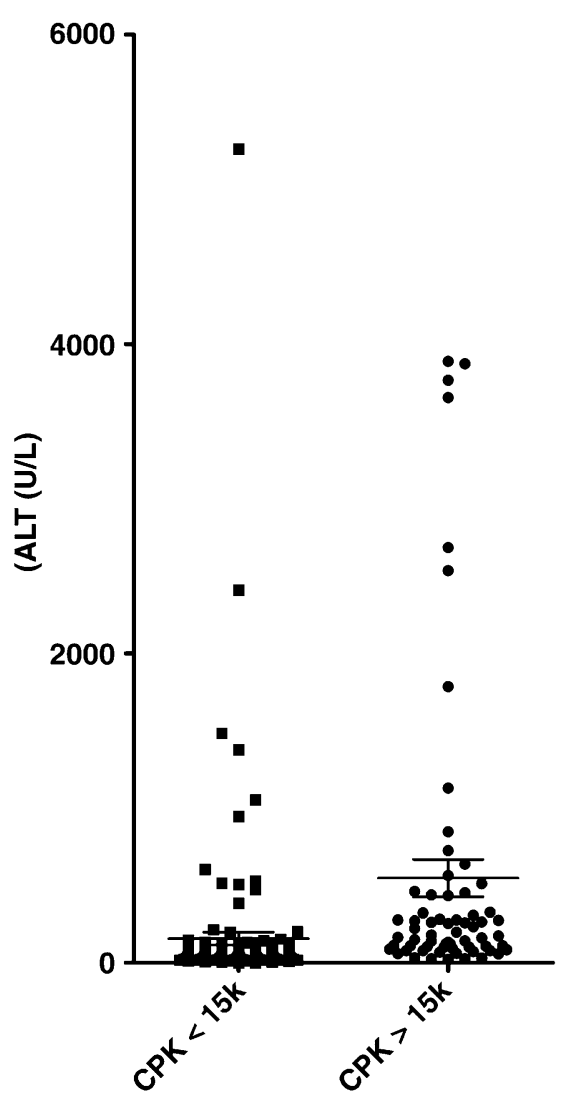

Fig. 5 Peak ALT values in all patients stratified according to peak CPK value

often associated with elevations of aminotransferases. In this study, we found that AST concentrations decrease in concert with CPK concentrations during hospitalization for rhabdomyolysis. This suggests that extrahepatic causes (i.e., skeletal muscle) may be a significant source of AST elevations in patients with rhabdomyolysis.

AST exists as two isoforms in humans: one localized to the cytosol of cells (roughly $20 \%$ of measured AST) and one localized to mitochondria $(80 \%)[10,11]$. The two isozymes have different kinetic properties and plasma clearances [10]. Most clinical laboratories do not differentiate between these isozymes. AST is located primarily in the liver, heart, skeletal muscle, kidney, brain, pancreas, lungs, leukocytes, and erythrocytes, in decreasing order of concentration [11, 12]. Plasma clearance of AST is performed primarily within hepatocytes $[13,14]$ and sinusoidal cells [10].

In contrast, ALT is a cytosolic enzyme isolated mostly to the liver [11]. As with AST, virtually no ALT is excreted in urine, and only a minute amount is found in bile [12]. Unlike AST, plasma clearance of ALT is effected by hepatocytes with no apparent contribution by sinusoidal cells [10].

It is generally accepted that ALT is a more specific marker of hepatic injury [15]. However, there is evidence of 
Fig. 6 Correlation between peak $\mathrm{CPK}$ and peak serum creatinine

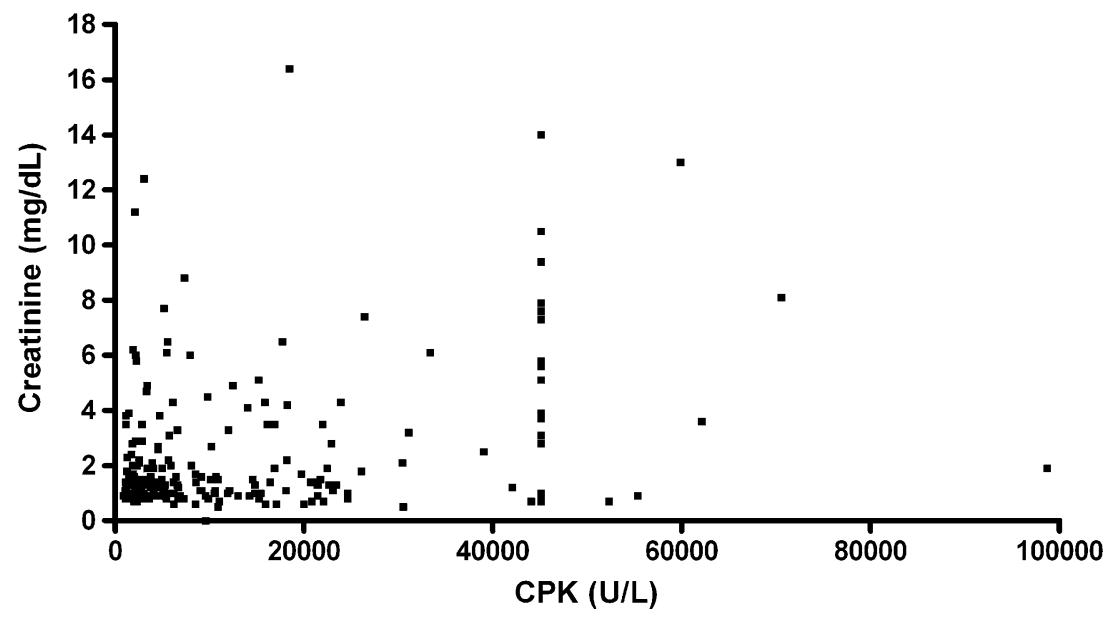

ALT production outside of the liver as demonstrated in a study from 1958 [16]. This study showed that overall, ALT exists at lower concentrations in all organs studied compared to AST. For instance, there was ten times as much ALT in the liver as compared to skeletal muscle. Similarly, cardiac tissue contained only $15 \%$ of the ALT concentration found in the liver. Interestingly, the concentration of ALT found in the kidney is $40 \%$ of what is seen in the liver. However, we would not expect this to contribute significantly in rhabdomyolysis given that the renal injury is due initially to tubular dysfunction rather than an ischemic insult.

It may be intuitive that AST would rise with rhabdomyolysis. A small study done by Nathwani et al. supports this [17]. Over a 27-year period, they identified 16 patients who had elevated aminotransferases following muscle injury, and in all cases, AST elevations were greater than ALT elevations [17]. In clinical practice, it is often unclear as to the source of elevated aminotransferases, particularly in the setting of rhabdomyolysis, and as such it may lead to inappropriate or dangerous testing. Korones et al. described two cases of children with elevated aminotransferases who underwent extensive testing, including liver biopsy. No liver disease was identified, and they were ultimately diagnosed with forms of muscular dystrophy after elevations in CPK were found [18]. It is important to bear in mind that while our data demonstrate aminotransferases (particularly AST) are increased in the setting of rhabdomyolysis, there is no means to determine if an observed AST or ALT is solely due to the rhabdomyolysis.

Although there appears to be some consensus that $1,000 \mathrm{U} / \mathrm{L}$ defines rhabdomyolysis [2, 5], there is no consensus as to what serum concentration constitutes significant rhabdomyolysis. Some authors have stated that a $\mathrm{CPK}>10,000 \mathrm{U} / \mathrm{L}$ is a marker of clinically significant rhabdomyolysis [19], while others believe that significant muscle injury and other end organ effects occur at levels as low as 5,000 U/L [20]. However, our goal in this study was not to determine what other clinical effects are caused by the rhabdomyolysis, but rather to determine the incidence and relationship between CPK and aminotransferase abnormalities with rhabdomyolysis.

Because some patients with rhabdomyolysis develop multiorgan failure (including liver failure), we chose to follow INR as a marker of significant liver injury. We chose the INR because both AST and ALT elevations correlate poorly with the degree of liver cell damage [21], although ALT concentrations are more specific for the liver [22]. In our patients, the mean INR for patients not on Coumadin during the first 2 days of hospitalization was just 1.2. Thus, the mild to moderately elevated aminotransferases seen in our patients with rhabdomyolysis were unlikely due to prior or concomitant significant liver injury, although some contribution of shock liver in the most critically ill patients is likely. This confounder is more clearly evident when looking at the subset of patients with the highest peak ALT (data not shown). Of six patients with the highest peak ALTs (mean 3,854 U/L; range 2,684-5,250), four died or suffered a cardiac arrest within 2 days of hospitalization despite modest degrees of rhabdomyolysis (mean 20,790 U/L; range $1,838-45,100)$.

Our aminotransferase data could be further skewed by contributions from additional confounders such as ethanol use. This could be relevant because the ratio of AST/ALT seen with alcohol use is typically greater than 2.0 [23]. In fact, in the setting of elevated AST and ALT, if the AST/ ALT ratio is greater than $2.0,90 \%$ of the time it is due to alcohol [24]. The population in Cohen's study was limited to those with liver disease confirmed with histopathology [24], whereas most of the patient with rhabdomyolysis will not fit that criteria. However, the aminotransferase elevations seen with alcohol abuse decrease over a period of weeks following cessation $[23,25]$, not over a period of 34 days as we found. Furthermore, our patient population is 
likely more heterogenous than the group used to generate the AST/ALT ratio [24]. Therefore, we feel that alcohol use is unlikely a significant confounder in this study. In addition, at least one previous study found that coffee ingestion was a better predictor of serum ALT than was alcohol [26].

In summary, we found that in the setting of rhabdomyolysis, elevated AST is common (occurring in $93 \%$ of patients) and serial serum AST concentrations fall in parallel to CPK concentrations. Furthermore, there is a trend toward peak AST concentrations increasing linearly with peak CPK concentrations. In contrast, ALT elevation is less common (occurring in $75 \%$ of patients), does not fall as rapidly as AST, and does not fall in parallel with peak CPK. Therefore, in the context of rhabdomyolysis, abnormal aminotransferases may not be indicative of hepatic injury.

\section{References}

1. Better OS (1990) The crush syndrome revisited (1940-1990). Nephron 55(2):97-103

2. Fernandez WG, Hung O, Bruno GR et al (2005) Factors predictive of acute renal failure and need for hemodialysis among ED patients with rhabdomyolysis. Am J Emerg Med 23(1):1-7

3. Goldfarb DS, Chung S (2002) The absence of rhabdomyolysisinduced renal failure the World Trade Center collapse. Am J Med 113:250

4. Sinert R, Kohl L, Rainone $\mathrm{T}$ et al (1994) Exercise-induced rhabdomyolysis. Ann Emerg Med 23:1301-1306

5. Ward MM (1988) Factors predictive of acute renal failure in rhabdomyolysis. Arch Intern Med 148:1553-1557

6. Gilbert EH, Lowenstein SR, Koziol-McLain J et al (1996) Chart reviews in emergency medicine research: where are the methods? Ann Emerg Med 27(3):305-308

7. Strassburg CP (2003) Gastrointestinal disorders of the critically ill. Shock liver. Best Pract Res Clin Gastroenterol 17(3):369-381

8. Calderon-Margalit R, Mor-Yosef S, Mayer M et al (2005) An administrative intervention to improve the utilization of laboratory tests within a university hospital. Int J Qual Health Care 17:243-248

9. Tarpey J, Lawler PG (1990) Iatrogenic anemia? A survey of venesection in patients in the intensive therapy unit. Anaesthesia $45: 396-398$
10. Kamimoto Y, Horiuchi S, Tanase S et al (1985) Plasma clearance of intravenously injected aspartate aminotransferase isozymes: evidence for preferential uptake by sinusoidal liver cells. Hepatology 5(3):367-375, Baltimore, Md. May-Jun

11. Goessling W, Friedman LS (2005) Increased liver chemistry in an asymptomatic patient. Clin Gastroenterol Hepatol 3(9):852-858

12. Schiff ER, Sorrell MF, Maddrey WC et al (2006) Schiff's diseases of the liver. Lippincott Williams \& Wilkins, Philadelphia

13. Wakim KG, Fleisher GA (1963) The fate of enzymes in body fluids - an experimental study. II. Disappearance rates of glutamic-oxalacetic transaminase I under various conditions. J Lab Clin Med 61:86-97

14. Fleisher GA, Wakim KG (1963) The fate of enzymes in body fluids - an experimental study. I. Disappearance rates of glutamicpyruvic transaminase under various conditions. J Lab Clin Med 61:76-85

15. Giannini EG, Testa R, Savarino V (2005) Liver enzyme alteration: a guide for clinicians. CMAJ 172(3):367-379

16. Wroblewski $F$ (1958) The clinical significance of alterations in transaminase activities of serum and other body fluids. Adv Clin Chem 1(2):313-351

17. Nathwani RA, Pais S, Reynolds TB et al (2005) Serum alanine aminotransferase in skeletal muscle diseases. Hepatology 41 (2):380-382, Baltimore, Md. Feb

18. Korones DN, Brown MR, Palis J (2001) "Liver function tests" are not always tests of liver function. Am J Hematol 66(1):46-48

19. Huerta-Alardin AL, Varon J, Marik PE (2005) Bench-to-bedside review: rhabdomyolysis - an overview for clinicians. Crit Care 9:158-169

20. Brown CV, Rhee P, Chan L et al (2004) Preventing renal failure in patients with rhabdomyolysis: do bicarbonate and mannitol make a difference? J Trauma 56:1191-1196

21. Pratt D, Kaplan MM (1999) Laborator tests. In: Schiff ER, Sorrell MF, Maddrey WC (eds) Schiff's Diseases f the Liver. LippincottRaven, Philadelphia, pp 205-244

22. Pratt D, Kaplan MM (2000) Evaluation of abnormal liver-enzyme results in asymptomatic patients. N Engl J Med 342:1266-1271

23. Conigrave KM, Davies P, Haber P et al (2003) Traditional markers of excessive alcohol use. Addiction 98(2):31-43, Abingdon, England

24. Cohen JA, Kaplan MM (1979) The SGOP/SGPT ratio-an indicator of alcoholic liver disease. Dig Dis Sci 24:835-838

25. Conigrave KM, Degenhardt LJ, Whitfield JB et al (2002) CDT, GGT, and AST as markers of alcohol use: the WHO/ISBRA collaborative project. Alcohol Clin Exp Res 26(3):332-339

26. Casiglia E, Spolaore P, Ginocchio G et al (1993) Unexpected effects of coffee consumption on liver enzymes. Eur J Epidemiol 9 (3):293-297 